\title{
Article \\ A Retrospective Study of the Proportion of Women at High and Low Risk of Intrauterine Infection Meeting Sepsis Criteria
}

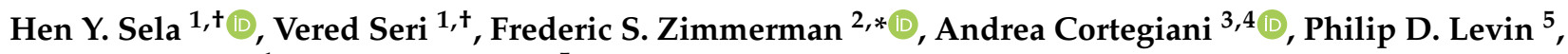 \\ Arnon Smueloff ${ }^{1}$ and Sharon Einav ${ }^{5}$ \\ 1 Shaare Zedek Medical Center, Department of Obstetrics and Gynecology, Hebrew University Faculty of \\ Medicine, Jerusalem 9103102, Israel; hysela@szmc.org.il (H.Y.S.); veredsery24@gmail.com (V.S.); \\ asamueloff@szmc.org.il (A.S.) \\ 2 Barzilai Medical Center, Department of Intensive Care, Ashkelon 7830604, Israel \\ 3 Department of Surgical, Oncological and Oral Science (Di.Chir.On.S.), University of Palermo, 90133 Palermo, \\ Italy; andrea.cortegiani@unipa.it \\ 4 Department of Anaesthesia, Intensive Care and Emergency, Policlinico Paolo Giaccone, 90127 Palermo, Italy \\ 5 Intensive Care Unit, Shaare Zedek Medical Center, Hebrew University Faculty of Medicine, \\ Jerusalem 9103102, Israel; levinp@szmc.org.il (P.D.L.); einav_s@szmc.org.il (S.E.) \\ * Correspondence: shmuelz@bmc.gov.il; Tel.: +972-8-674-3322 \\ + These authors contributed equally to the manuscript.
}

Citation: Sela, H.Y.; Seri, V.;

Zimmerman, F.S.; Cortegiani, A.;

Levin, P.D.; Smueloff, A.; Einav, S

A Retrospective Study of the

Proportion of Women at High and

Low Risk of Intrauterine Infection

Meeting Sepsis Criteria.

Microorganisms 2022, 10, 82. https://

doi.org/10.3390/microorganisms

10010082

Academic Editor:

Sofia Costa-de-Oliveira

Received: 16 November 2021

Accepted: 28 December 2021

Published: 31 December 2021

Publisher's Note: MDPI stays neutral with regard to jurisdictional claims in published maps and institutional affiliations.

Copyright: () 2021 by the authors. Licensee MDPI, Basel, Switzerland. This article is an open access article distributed under the terms and conditions of the Creative Commons Attribution (CC BY) license (https:// creativecommons.org/licenses/by/ $4.0 /)$.

\begin{abstract}
The Surviving Sepsis Campaign recently recommended that qSOFA not be used as a single parameter for identification of sepsis. Thus, we evaluated the efficacy of SIRS and qSOFA scores in identifying intrauterine infection. This case-control study evaluates SIRS and qSOFA criteria fulfillment in preterm premature rupture of membranes $(n=453)$-at high infection risk—versus elective cesarean-at low infection risk $(n=2004)$; secondary outcomes included intrauterine infection and positive culture rates. At admission, $14.8 \%$ of the study group and $4.6 \%$ of control met SIRS criteria $(p=0.001)$, as did $12.5 \%$ and $5.5 \%$ on post-operation day (POD) $1(p=0.001)$, with no significant differences on POD 0 or 2. Medical records did not suffice for qSOFA calculation. In the study group, more cultures (29.8\% versus $1.9 \%$ - cervix; $27.4 \%$ versus $1.1 \%$ - placenta; $7.5 \%$ versus $1.7 \%$-blood; $p=0.001$-all differences) and positive cultures (5.5\% versus $3.0 \%$-urine $-p=0.008 ; 4.2 \%$ versus $0.2 \%$ - cervix $-p=0.001 ; 7.3 \%$ versus $0.0 \%$-placenta $-p=0.001 ; 0.9 \%$ versus $0.1 \%$ - blood $-p=0.008$ ) were obtained. Overall, $10.6 \%$ of the study group and $0.4 \%$ of control met the intrauterine infection criteria $(p=0.001)$. Though a significant difference was noted in SIRS criteria fulfillment in the study group versus control, there was considerable between-group overlap, questioning the utility of SIRS in intrauterine infection diagnosis. Furthermore, the qSOFA scores could not be assessed.
\end{abstract}

Keywords: chorioamnionitis; intrauterine infection; SIRS; sepsis; early identification

\section{Introduction}

The Surviving Sepsis guidelines mandate the investment of a concerted effort towards the early recognition of severe infection [1]. The use of early warning scores has been associated with earlier treatment and improved mortality in patients with suspected sepsis [2]. Such scores therefore constitute an important part of the assessment of patients with suspected infection. Pregnant women are at increased risk for certain types of infection. In fact, infection is the third most common cause of maternal death [3-5]. Yet, the vital signs and laboratory values of pregnant women not only differ from those of the non-pregnant population, but also change with gestational age, which raises questions regarding the value of scores currently used to identify severe infection in the pregnant population.

Several generic scoring systems, including the Modified Early Obstetric Warning System, have been proposed to facilitate early recognition of critical illness, including sepsis, in obstetric patients [6-8]. However, these tools are inherently non-specific. The tools used 
specifically to identify sepsis include the systemic inflammatory response syndromes (SIRS) criteria, sequential organ failure assessment (SOFA) and quick- SOFA (qSOFA) criteria. The SIRS criteria were first recommended for early identification of severe infection $[9,10]$, mostly for the purpose of defining target populations for sepsis studies. In 2016, based on new research, a recommendation was put forward to replace the SIRS criteria with the qSOFA criteria in order to more effectively identify patients outside of the critical care setting who are at high risk of mortality. The qSOFA criteria, which reflect the severity of specific organ failures rather than the immune response to the presence of infection, were proposed to be not only an evidence-based means of recognizing early infection but also clinically more practical [11]. However, recent recommendations have rejected the qSOFA as a single screening tool, due to insufficient sensitivity [12].

Genitourinary infections are very common in pregnant women. One of the more common infections of this type occurring in pregnancy and the peripuerum is intrauterine infection or inflammation (previously known as chorioamnionitis [13]). Preterm premature rupture of membranes (PPROM) is especially associated with an increased risk of intrauterine infection; at least 10\% of women with PPROM develop intrauterine infection [14-16]. In low risk obstetric populations this risk is much lower and is reported to be between $1.7 \%$ and $5 \%[17,18]$ Such infections can result in maternal morbidity and mortality and are accompanied by a high rate of fetal loss. Early broad spectrum antibiotic treatment of intrauterine infection may reduce maternal and fetal morbidity and mortality $[8,13]$. Thus, the early identification of severe infection is vital for reducing the morbidity associated with this condition. This current study investigated the value of various tools, currently and previously recommended for identifying the presence of sepsis in the general population, in identification of intrauterine infection. The primary objective was to evaluate the rate of SIRS and qSOFA criteria fulfillment among peripartum women with a higher likelihood of infection versus those at low risk. The secondary objectives were (a) to evaluate the frequency that the components of these scores are actually available for scoring in the peripartum population and (b) the rate of documented intrauterine infection and the rate of positive cultures (urine, cervix, placenta and blood) in the two groups.

\section{Materials and Methods}

The study protocol was submitted to the IRB committee and given its retrospective nature, the IRB approved the study with waiver of informed consent (approval number: SZMC-0001-16). Following approval, a retrospective case-control study was conducted on all women who underwent cesarean delivery (CD) during a 10 year period in the Shaare Zedek Medical Center (SZMC), Jerusalem, Israel.

Clinical setting: The SZMC is a 1000-bed university-affiliated acute care hospital with a Division of Obstetrics that includes a high risk pregnancy unit, two delivery suites with attached dedicated obstetric operating rooms and five maternity wards. The annual rate of admissions for delivery approximated 14,000 during the study period.

Inclusion exclusion criteria: The study group consisted of women admitted to hospital with a diagnosis of PPROM at a gestational age of 24 through 36 weeks, provided they underwent $C D$ within 7 days of admission and prior to week 37. Previous studies have shown that these women have a $10 \%$ risk of developing intrauterine infection or inflammation [14]. The control group consisted of subjects undergoing CD at a gestational age of 37 weeks or more, with no rupture of membranes and no trial of labor prior to surgery. In women with these characteristics the risk of intrauterine infection or inflammation is considered approximately $1.7 \%[17,18]$, but there is scarce data of good quality on the topic. Women with a vaginal delivery, those with rupture of membranes prior to week 24 and those with rupture of membranes or trial of labor who gave birth in week 37 or later were excluded from both groups. Additionally, women with more than 7 days elapsing from rupture of membranes to delivery were excluded.

Case identification: Relevant cases admitted to the SZMC (August 2005-December 2015) were identified via structured queries to the obstetric electronic medical record (NeSS 
Technologies, Israel). For the study group, the query first identified all women admitted to the SZMC between August 2005 and December 2015 and then selected those who (1) had undergone CD prior to week 37 and within one week of admission and (2) had an admission or discharge diagnosis of rupture of membranes. If a discrepancy was found between the admission and discharge diagnosis, the medical record was reviewed. In such cases, if rupture of membranes had not been recorded on admission or throughout hospitalization - the case was removed from the study. Most relevant data were available in the NeSS-EMR for the cases identified for this study. However, if required, missing data were also completed from hard copy admission files.

For the control group, the query identified women who had undergone $\mathrm{CD}$ at a gestational age of $37-40$ weeks with no trial of labor. In this group there was a large quantity of eligible subjects. Therefore, those for whom full data (as described below) was not available via the NESS-EMR were excluded and no review of the hard copy admission files was required.

Variables: The primary outcome measures were (1) the rate of SIRS and qSOFA criteria fulfillment among the study population and (2) the difference in the rates of SIRS and qSOFA criteria fulfillment in the study population as compared to the control population. Secondary outcome measures included (a) the frequency that the components of above scores were actually available the peripartum population and (b) the rate of documented intrauterine infection and positive cultures (urine, cervix, placenta and blood) in the two groups.

In order to study these outcomes, the data to be collected included the vital signs (heart rate, blood pressure, temperature, respiratory rate) and mental status documented in real time by the nursing staff, as well as complete blood count (CBC), creatinine and bilirubin levels on the day of admission and on POD 0, 1 and 2 and all culture results.

For purposes of this study, women were considered likely to have intrauterine infection if their discharge notes included a relevant diagnostic code (ICD-9 658.41) or if they had been treated with broad spectrum antibiotics within $24 \mathrm{~h}$ of their delivery, these cases were reviewed to ascertain the diagnosis of intrauterine infection. Therefore, the data collected also included the presence of relevant coding and any evidence in the file that the woman had been treated with broad-spectrum antibiotics. During the study years the clinical criteria used for diagnosing intrauterine infection were maternal fever $>38^{\circ} \mathrm{C}$ along with one or more of the following: maternal or fetal tachycardia, elevated maternal white blood cell count, uterine tenderness and purulent fluid or purulent discharge from the cervical os. We did not use the CDC recommendation of four days of continuous antibiotic usage to identify sepsis, as we did not collect length of treatment because our institutional protocol is to continue antibiotics after CD until either at least $24 \mathrm{~h}$ have elapsed without fever or until the return of cultures. In women who deliver vaginally, treatment is continued for at least $24 \mathrm{~h}$ post-partum; if there are additional risk factors, antibiotic treatment is continued for at least $24 \mathrm{~h}$ afebrile or until return of cultures.

Potential sources of bias: During data collection several issues arose. It was discovered that temperature was not recorded on POD 0 for $88 / 453$ (19.4\%) of subjects in the study group and 900/2004 (44.9\%) of subjects in the control group. Upon review it was apparent that these omissions were in subjects undergoing an evening operation and arriving in the maternity ward close to midnight. Since these cases comprised a considerable percentage of our study population and the time of omission was consistent, concerns arose regarding potential documentation bias. This was addressed in the analysis by comparing the women with missing data to those without missing data (see Table S1). The proportion of documentation omissions with regard to blood pressure, heart rate and temperature excluding POD0 was negligible and random, therefore women lacking data regarding any of these were excluded from the study (see Table S2). An additional major issue that became apparent during data collection was the paucity of documentation of the respiratory status and mental status of women in both the control and the study groups. This deficit precluded calculation of the subjects' qSOFA scores. 
Method of data collection: The SZMC has separate electronic medical records (EMRs) for obstetrical and all other patient data. During the study period, the obstetric ward was also performing duplicate documentation as changes were being implemented to the EMR for accreditation purposes. Cases were identified via the NESS-EMR, and the vital signs were collected from the NESS-EMR (which was in use in the various obstetric departments). The rest of the data were collected from the hospital-wide EMR. As noted above, when data were unavailable in the EMR, hard copy files were also reviewed to seek additional information.

For all women, the initial set of data collected included the variables recorded at the time of admission regardless of the location of admission. For women who were hospitalized but did not undergo CD immediately upon admission, data were collected from the period they were observed in the high risk pregnancy unit. Women who remained in the delivery suite more than $24 \mathrm{~h}$ before CD had data collected from there. All data collected from the period after CD were taken from maternity ward notes.

Sample size considerations: The study was designed as a 1:4 case-control study. Based on the data in the literature, we hypothesized that in the study group, the rate of intrauterine infection would be approximately $10 \%$ [14-16,19], whereas in the control group this rate would be at least $1.7 \%$ and no more than $5 \%[17,18]$. According to these data, 437 cases in the study group and 1748 cases in the control group were needed to reject (with a power of 0.95) the null hypothesis that there was an identical rate of fulfillment of sepsis criteria in the study and control groups. Upon initial review of our computerized database, we identified approximately 600 women with a diagnosis of PPROM. We assumed that about $30 \%$ of these would be eliminated after manual review. Thus 420 would remain in the study group, of whom approximately $42(10 \%)$ would be found to have an intrauterine infection. No more than 2000 subjects were required in the control group, of whom no more than 100 (5\%) would be found to have an intrauterine infection. The probability of a type I error associated with this test of the null hypothesis was calculated as 0.05 .

Quantitative variables: The lowest systolic blood pressure and highest pulse and fever recorded each day were used for analysis. The plan was to describe the Glasgow Coma Scale as the total sum rather than its three components. Laboratory data, including leukocyte and platelet count, creatinine and bilirubin, were collected and analyzed as presented by the hospital laboratory.

Data management and statistical analysis: The data were downloaded from the EMR to a Microsoft Excel (Ver. 2010) database and then transferred to SPSS (IBM Corp. Released 2013. IBM SPSS Statistics for Windows, Version 22.0. Armonk, NY, USA, IBM Corp.), which was used for analysis. All data from hard copy patient admission files were manually added to the original file.

In the first step, descriptive statistics (i.e., numbers, proportion and means) were used to describe the study population as a whole and study and control group characteristics (Table 1).

In the second step, comparisons between women with and without missing data were performed and in the third step, comparisons between the study and control groups were performed. In both of these steps, comparison between proportions was performed using the $\chi$ 2-score (e.g., for demographic and clinical features) or the Fisher's exact test (e.g., for the rate of positive cultures). To compare continuous variables the Student's $t$-test (e.g., for maternal age) or the Mann-Whitney-Wilcoxon test (e.g., for vital signs and obstetric characteristics such as number of gestations and previous CDs) were used. In all tests, two-tailed $p$-values were taken and a $p$-value $<0.05$ was considered significant.

Finally, we performed sensitivity analyses to compare the rate of positive urinary cultures between the two groups, once by assuming that the missing cultures were positive and once by assuming they were negative. 
Table 1. Demographic, obstetric and medical characteristics of study group versus control group. PPROM: preterm premature rupture of membranes; CI: confidence interval.

\begin{tabular}{lccc}
\hline Characteristic & PPROM $\boldsymbol{n}=\mathbf{4 5 3}(\mathbf{\%})$ & Control $\boldsymbol{n}=\mathbf{2 0 0 4}(\mathbf{\%})$ & $\boldsymbol{p}$ \\
\hline Maternal age (mean $\pm \mathrm{CI})$ & $31.5 \pm 6.7$ & $33.8 \pm 5.3$ & 0.001 \\
$\quad>35$ year & $127(28.0)$ & $750(37.4)$ & 0.001 \\
Member of minority & $70(15.5)$ & $220(11.0)$ & 0.001 \\
Completed secondary education & $411(90.7)$ & $1934(96.5)$ & 0.005 \\
\hline Gestation number & $3.2 \pm 2.5$ & $3.9 \pm 2.6$ & 0.001 \\
Previous cesareans (mean $\pm \mathrm{CI})$ & $0.4 \pm 0.8$ & $1.1 \pm 1.2$ & 0.001 \\
$\quad$ Any previous cesarean & $121(26.7)$ & $1077(59.9)$ & 0.001 \\
Previous abortion $($ mean $\pm \mathrm{CI})$ & $0.7 \pm 1.3$ & $0.7 \pm 1.1$ & 0.531 \\
In vitro fertilization & $126(27.8)$ & $319(15.9)$ & 0.001 \\
Twin pregnancy & $160(35.3)$ & $200(10.0)$ & 0.001 \\
\hline Gestational diabetes & $46(10.2)$ & $269(13.4)$ & 0.060 \\
Hypertension & $18(4.0)$ & $69(3.4)$ & 0.581 \\
Hypothyroidism & $29(6.4)$ & $117(5.8)$ & 0.647 \\
\hline
\end{tabular}

\section{Results}

Of the 142,372 admissions for delivery that took place in the SZMC during the study period, 7499 deliveries occurred at a gestational age of 24-36 weeks. Among these, 621 women were identified as having a presumptive diagnosis of PPROM and also underwent CD. After chart review, 168 were excluded as they did not fulfill the study group criteria in full. Likewise, during the study period 107,401 deliveries took place at a gestational age of 37-40 weeks. Among these, 3159 women underwent CD without a trial of labor. After chart review, 1155 were excluded as they did not meet the overall control group criteria. In total, 453 subjects were included in the study group and 2004 subjects were included in the control group (Figure 1).

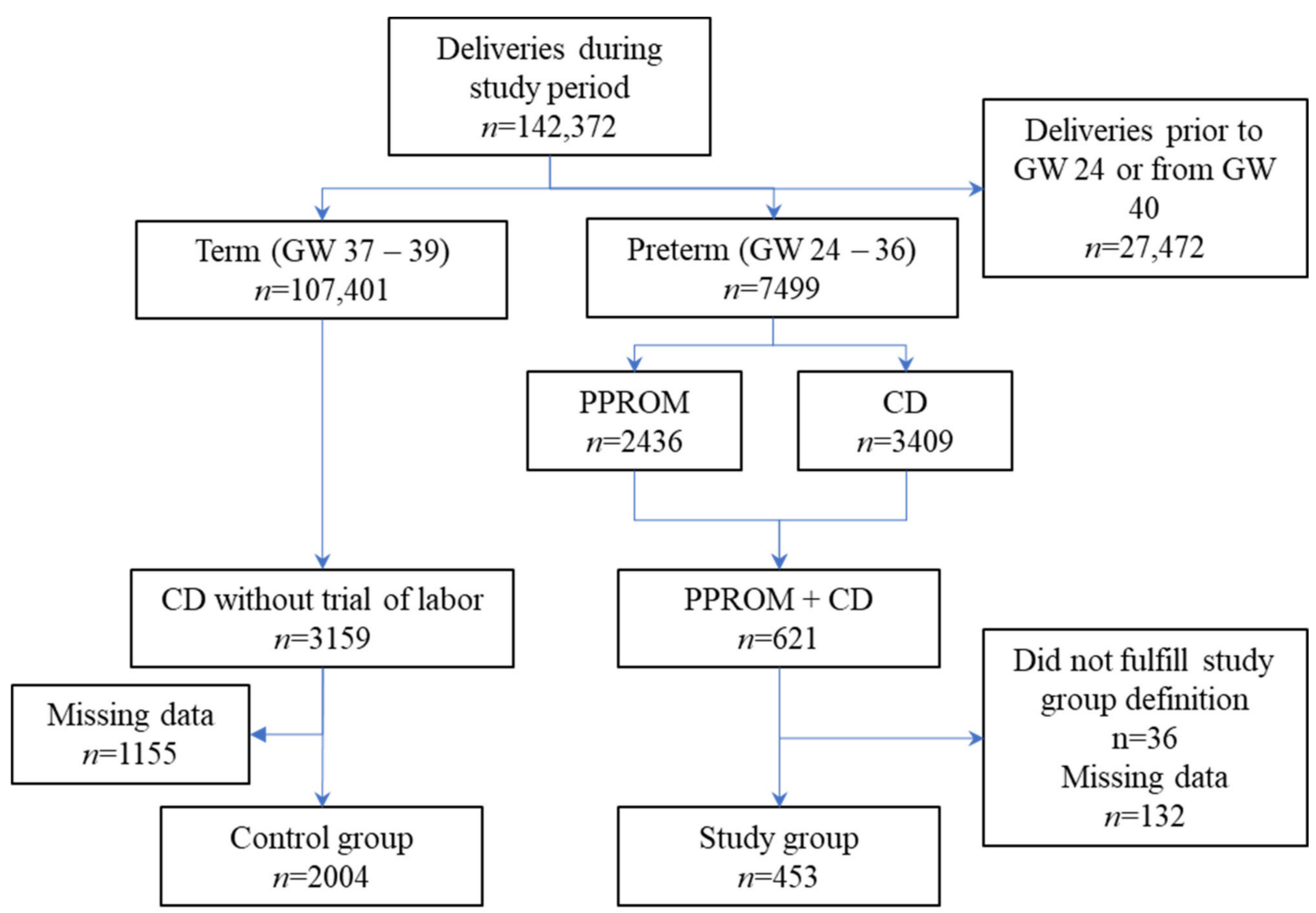

Figure 1. Study inclusion/exclusion process. GW: gestational week; CD: cesarean delivery; PPROM: preterm premature rupture of membranes. 


\subsection{Assessment of Reporting Bias}

The results of the comparison of cases with missing data to those with no missing data are presented in Table S1. In the control group, patients with missing data were slightly younger, had fewer previous pregnancies, had undergone fewer CDs and had a higher incidence of hypertension than those without missing data. No significant differences were observed in the study group.

\subsection{Description of the Study Population}

The average age of the women was $33.6 \pm 5.6$ years and they had undergone $3.8 \pm 2.6$ previous deliveries and $1.1 \pm 0.9$ previous caesarean deliveries. The women in the study group were younger than those in the control group. They were also more likely to be a member of a minority group and were less likely to have completed secondary education. The women in the study group also had fewer deliveries and caesarean deliveries than those in the control group (Table 1).

\subsection{Comparison between Study and Control Groups - Vital Signs and Complete Blood Count}

The women in study group had a higher body temperature than the women in the control group at the time of admission and on POD $0(36.73 \pm 0.4$ versus $36.68 \pm 0.3$, $p=0.005$; and $36.72 \pm 0.5$ versus $36.46 \pm 0.5, p=0.001$, respectively). They also had a higher mean arterial pressures and leukocyte counts throughout admission. Although these differences were all statistically significant, they were clinically meaningless. Women in the study group also had higher heart rates throughout admission, but the statistical significance of this finding varied by day (Table 2). No significant difference in platelet count between the two groups were noted throughout the admission.

Table 2. Vital signs and white cell counts of study group versus control group. PPROM: preterm premature rupture of membranes; POD: post-operation day.

\begin{tabular}{lcccccc}
\hline Characteristic & DAY & PPROM & $n$ & Control & $n$ & $p$ \\
\hline \multirow{4}{*}{ Temperature } & Admission & $36.73 \pm 0.4$ & 453 & $36.68 \pm 0.3$ & 2004 & 0.005 \\
& POD0 & $36.72 \pm 0.5$ & 365 & $36.46 \pm 0.5$ & 1104 & 0.001 \\
& POD1 & $36.82 \pm 0.5$ & 453 & $36.81 \pm 0.4$ & 2004 & 0.367 \\
& POD2 & $36.69 \pm 0.4$ & 453 & $36.65 \pm 0.4$ & 2004 & 0.147 \\
\hline \multirow{5}{*}{ Pulse } & Admission & $92.7 \pm 14.4$ & 453 & $88.2 \pm 11.4$ & 2004 & 0.001 \\
& POD0 & $84.8 \pm 12.5$ & 453 & $83.2 \pm 10.8$ & 2004 & 0.06 \\
& POD1 & $90.6 \pm 11.5$ & 453 & $88.8 \pm 9.2$ & 2004 & 0.029 \\
Mean arterial & POD2 & $88.9 \pm 11$ & 453 & $87.8 \pm 9.6$ & 2004 & 0.34 \\
pressure & Admission & $90.3 \pm 10.9$ & 453 & $85.6 \pm 9.3$ & 2004 & 0.001 \\
& POD0 & $76.8 \pm 10.5$ & 453 & $74.3 \pm 9.5$ & 2004 & 0.001 \\
& POD1 & $73.6 \pm 9.5$ & 453 & $70.8 \pm 8.2$ & 2004 & 0.001 \\
Leukocyte count & POD2 & $78.2 \pm 10$ & 453 & $76.8 \pm 9.1$ & 2004 & 0.008 \\
$\left(\times 10^{3}\right)$ & Admission & $10.3 \pm 9.2$ & 452 & $9.2 \pm 2.2$ & 1997 & 0.001 \\
& POD0 & $13.4 \pm 11.7$ & 310 & $11.7 \pm 3.3$ & 1617 & 0.001 \\
& POD1 & $12.2 \pm 11.4$ & 187 & $11.4 \pm 3$ & 587 & 0.007 \\
& POD2 & $11.4 \pm 9.7$ & 56 & $9.7 \pm 2.6$ & 138 & 0.005 \\
\hline
\end{tabular}

\subsection{Rates of Vital Sign Documentation}

The documentation rates of vital sign recordings and complete blood counts were high $(>98 \%)$ in both groups. No significant difference in documentation rates of these variables was noted between the groups.

\subsection{Comparison between Study and Control Groups-Fulfillment of SIRS and qSOFA Criteria}

Overall, $14.8 \%(67 / 453)$ of the women in the study group and $4.6 \%(92 / 2004)$ the women in the control group fulfilled SIRS criteria at admission $(p=0.001)$. Likewise, on POD1 $12.5 \%(57 / 453)$ of the women in the study group and 5.5\% $(110 / 2004)$ of the women 
in the control group fulfilled SIRS criteria $(p=0.001)$. No significant differences in the rate of fulfillment of SIRS criteria were noted on other hospitalization days (Figure 2). The data in the medical records did not suffice for the calculation of qSOFA scores.

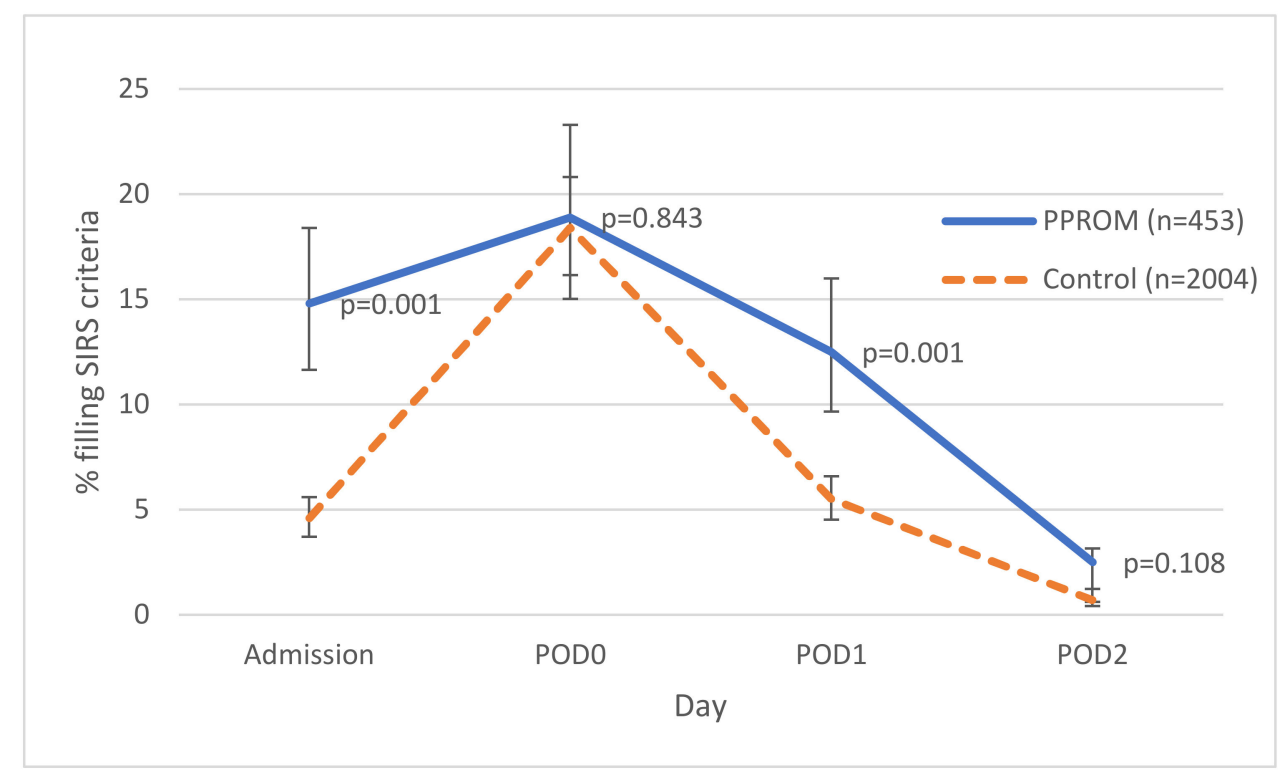

Figure 2. Proportion of patients that fulfilled the systemic inflammatory response syndrome (SIRS) criteria among the study and control groups. POD: post-operative day; PPROM: preterm premature rupture of membranes.

\subsection{Comparison between Study and Control Groups-Rate of Diagnosis of Intrauterine Infection}

Among the study group 48/453 (10.6\%) of the women, and 8/2004 (0.4\%) of the women among the control group met the criteria used to define the presence of intrauterine infection $(p=0.001)$.

\subsection{Comparison between Study and Control Groups-Cultures}

\subsubsection{Sampling Rates}

Urine cultures were obtained from $91.4 \%(414 / 453)$ of women in the study group and $100.0 \%(2004 / 2004)$ of those in the control group $(p=0.001)$. The proportion of women from whom cervical, placental and blood cultures were obtained was considerably lower in both groups. However, significantly more cultures of any kind were obtained from the study group than from the control group-cervix: 135/453 (29.8\%) versus 38/2004 (1.9\%), placenta: $124 / 453(27.4 \%)$ versus $22 / 2004(1.1 \%)$, and blood: $34 / 453(7.5 \%)$ versus $34 / 2004$ $(1.7 \%)$, respectively $(p=0.001$ for all).

\subsubsection{Culture Results}

Among the cultures taken, there were significantly more positive cultures in the study group than in the control group; $25 / 453(5.5 \%)$ versus $60 / 2004$ (3.0\%) for urine cultures $(p=0.008), 19 / 453(4.2 \%)$ versus $4 / 2004(0.2 \%)$ for cervical cultures $(p=0.001), 33 / 453$ $(7.3 \%)$ versus $1 / 2004(0.0 \%)$ for placental cultures $(p=0.001)$ and $4 / 453(0.9 \%)$ versus $3 / 2004(0.1 \%)$ for blood cultures $(p=0.008)$ (Table 3). 
Table 3. Microbiological cultures—study group versus control group. PPROM: preterm premature rupture of membranes.

\begin{tabular}{ccccccc}
\hline & \multicolumn{3}{c}{ Cultures Obtained } & \multicolumn{3}{c}{ Positive Cultures } \\
\cline { 2 - 7 } Body Site & $\begin{array}{c}\text { PPROM } \\
(\boldsymbol{n}=\mathbf{4 5 3})\end{array}$ & $\begin{array}{c}\text { Control } \\
(\boldsymbol{n}=\mathbf{2 0 0 4 )}\end{array}$ & $\boldsymbol{p}$ & $\begin{array}{c}\text { PPROM } \\
(\boldsymbol{n}=\mathbf{4 5 3 )}\end{array}$ & $\begin{array}{c}\text { Control } \\
(\boldsymbol{n}=\mathbf{2 0 0 4 )})\end{array}$ & $\boldsymbol{p}$ \\
\hline Urine & $414(91.4)$ & $2004(100.0)$ & 0.001 & $25(5.5)$ & $60(3.0)$ & 0.008 \\
Cervix & $135(29.8)$ & $38(1.9)$ & 0.001 & $19(4.2)$ & $4(0.2)$ & 0.001 \\
Placenta & $124(27.4)$ & $22(1.1)$ & 0.001 & $33(7.3)$ & $1(0.0)$ & 0.001 \\
Blood & $34(7.5)$ & $34(1.7)$ & 0.001 & $4(0.9)$ & $3(0.1)$ & 0.008 \\
\hline
\end{tabular}

\subsubsection{Sensitivity Analysis-Urinary Cultures}

A sensitivity analysis was only performed for urinary cultures, as these were routinely obtained for most of the study population (whereas other cultures were only obtained when infection was suspected). When the analysis was performed with the assumption that the missing cultures would have been positive, the difference between the study group and the control group increased. When the analysis was performed with the assumption that the missing cultures were negative, the difference between the groups decreased but remained statistically significant (Table S3).

\section{Discussion}

Sepsis continues to be one of the leading causes of maternal death worldwide, including in the developed world [20]. Late diagnosis and treatment of sepsis has been shown to increase mortality in the general population [21] and an analysis of maternal deaths following sepsis also showed that late detection and treatment contributed to maternal mortality [22]. The risk of sepsis increases in women who undergo caesarean section during labor and those with PPROM [23]. Therefore, early diagnosis of severe acute infection is particularly important in these populations. This retrospective study of 2457 women was designed to examine the proportion of women meeting SIRS and qSOFA criteria among those hospitalized due to PPROM (a population at high risk of intrauterine infection) as compared to the proportion among those at low risk of intrauterine infection. Significantly more women in the high risk group met SIRS criteria than women in the low risk group, especially on admission. However, there were women in both groups that did not meet these criteria despite clear differences in risk and eventual culture positivity. In other words, pregnant/peripartum women with a high likelihood of severe systemic infection may not necessarily fulfill SIRS criteria while those fulfilling SIRS criteria may not really be at risk of systemic infection. These findings are similar to a previous retrospective study, which failed to show an association between SIRS criteria and risk for intensive care unit transfer, sepsis, or death among pregnant women with intrauterine infection [19]. However, unlike the previous study - we were able to evaluate SIRS criteria for the majority of patients included in both arms of the study (Figure 1). In addition, the study group was compared to a control group, and we were able to analyze the applicability of sepsis criteria to multiple points relative to $C D$.

A second important finding of this study is the extent of missing documentation and cultures in this population. This finding is not unique to our study [19] and offers further support for recent recommendations against the use of qSOFA as a single sepsis screening tool [12].

It should be noted that the rate of infection as identified in our study is similar to that of previous studies. Thus, in this study, as in previous studies [14-16,19], 10\% those with PPROMM developed intrauterine infection. Furthermore, despite the low overall rate of culture collection, the rates of positive urinary, vaginal and placental cultures observed in our study group were similar to those described in previous studies [14,24]. Additionally, our control group had rates of positive urinary cultures similar to those 
reported in previous studies [25-27]. These similarities increase the generalizability of findings to a larger obstetric population.

Our study results support findings from previous studies suggesting that the physiological changes of pregnancy complicate the identification of systemic infection in pregnant women at risk $[19,28]$. Though there are generally accepted normal ranges in pregnancy for the various components of the SIRS criteria, these are based on very little data. Current definitions of abnormal values in women with PPROM are based on expert consensus only [13]. This study demonstrated a statistically significant between-group difference in the proportion of those meeting SIRS criteria; this difference was maintained at several time points during admission. Nevertheless, because a substantial number of patients in the study group did not meet SIRS criteria, whereas a substantial number of those in the control group did, these criteria do not seem particularly useful for differentiating between women at risk and those not at risk.

As noted above a significant secondary finding is the extent of missing documentation and cultures. This finding may be related to long-standing midwifery traditions [29] and the tendency, as described in other areas [30], to treat the pregnancy rather than the woman carrying it. The qSOFA score was put forward as a tool for identifying sepsis in the general population precisely because its components are used to monitor patients on the ward. However, this assumption does not hold true in obstetric populations. This study and others $[29,31]$ highlight that the relevant maternal parameters (particularly respiratory rate and mental status) are not documented at all in many births. The question arises whether these simple measurements should be incorporated into the obstetric work routine in these departments.

Despite the similarity between the women in the two groups, more blood, placental and vaginal cultures were taken from those included in the study group. Had these been taken routinely from all women at risk, one could argue that the actual risk profile is associated with a higher rates of positive culture. However, these cultures were not taken routinely, which indicates that the treating physicians had higher suspicion of infection in some cases. These suspicions were later substantiated by an associated higher positive culture rate. In the absence of clear-cut differences between the cases, the question remains as to what led clinicians to suspect infection. If this suspicion can be quantified, it may be possible to establish clearer indicators for a prompt diagnosis.

This study has several limitations. Although this study did include a control arm, it is a retrospective data analysis of an unmatched cohort, and, therefore, its conclusions should be approached with caution. The need for caution is further reinforced by the demographic differences between the study and control groups (Table 1). A further limitation is the lack of full documentation, necessitating the elimination of a substantial percentage of the initial cohort, and making it impossible to evaluate qSOFA at all. In addition, the rate of cultures obtained was lower than expected; still, the percentage of positive cultures was higher in the study group than in the control group, with this difference remaining statistically significant in bi-directional sensitivity analyses for urinary cultures. Furthermore, due to study limitations, we were not able to use the CDC recommendation of 4 days of continuous antibiotic usage to identify sepsis in retrospective studies. Administrative data also has its limitations. Some women included in the study group did not have intrauterine infection but the rate of SIRS criteria fulfillment was still high. The use of administrative data to perform clinical research is a common practice, despite reliance on the accuracy of the codes used. Prior studies have shown that intrauterine infection codes have a negative predictive value of $98 \%$ and a positive predictive value of 50\% [32]. Finally, there are inflammatory markers that we did not examine. Although several studies have proposed that C-reactive protein (CRP), procalcitonin (PCT) and interleukin 6 (IL6) may be used for early identification of intrauterine infection [33,34], a recent systematic review of diagnostic test accuracy studies noted that there is insufficient evidence to support the use of these markers for diagnosing intrauterine infection in PPROM [35]. 


\section{Conclusions}

This study found that despite a statistically significant difference in the rate of SIRS in patients with PPROM undergoing CS versus those undergoing elective CS, there is still a considerable overlap between these two groups, thus calling into question the utility of SIRS in the prompt diagnosis of intrauterine infection in pregnant women and possibly in the immediate postpartum period. Additional studies are required to validate our preliminary findings that SIRS criteria should not be used for screening. Missing documentation relevant to the qSOFA score supports recent recommendations against the use of qSOFA as a single sepsis screening tool [12]. This study further emphasizes the need for better maternal monitoring in the peripartum period and a more widespread use of cultures in identifying intrauterine infection in at-risk populations.

Supplementary Materials: The following are available online at https:/ / www.mdpi.com/article / 10.3390/microorganisms10010082/s1, Table S1: Comparison of subjects included in study versus subjects excluded due to missing data, Table S2: Subjects with missing vital signs, Table S3: Sensitivity analysis-urinary cultures.

Author Contributions: Conceptualization, H.Y.S., V.S., P.D.L., A.S. and S.E.; Data curation, H.Y.S., V.S. and S.E.; Formal analysis, H.Y.S., V.S., F.S.Z., A.C. and S.E.; Investigation, H.Y.S., V.S., A.S. and S.E.; Methodology, H.Y.S., V.S., F.S.Z., P.D.L., A.S. and S.E.; Project administration, H.Y.S. and S.E.; Supervision, S.E.; Validation, H.Y.S.; Writing—original draft, H.Y.S., V.S., F.S.Z. and S.E.; Writingreview and editing, H.Y.S., F.S.Z., A.C. and S.E. All authors have read and agreed to the published version of the manuscript.

Funding: This research received no external funding.

Institutional Review Board Statement: The study was conducted in accordance with the Declaration of Helsinki, and approved by the Institutional Review Board of Shaare Zedek Medical Center (protocol code SZMC-0001-16, date of approval: 9 May 2016).

Informed Consent Statement: Patient consent was waived due to the retrospective nature of the study with de-identification of all patient data.

Data Availability Statement: The data presented in this study are available on request from the corresponding author. The data are not publicly available due to privacy concerns.

Conflicts of Interest: The authors declare no conflict of interest.

\section{References}

1. Levy, M.M.; Evans, L.E.; Rhodes, A. The Surviving Sepsis Campaign Bundle: 2018 update. Intensive Care Med. 2018, 44, 925-928. [CrossRef] [PubMed]

2. $\quad$ Levy, M.M.; Dellinger, R.P.; Townsend, S.R.; Linde-Zwirble, W.T.; Marshall, J.C.; Bion, J.; Schorr, C.; Artigas, A.; Ramsay, G.; Beale, R.; et al. The Surviving Sepsis Campaign: Results of an international guideline-based performance improvement program targeting severe sepsis. Intensive Care Med. 2010, 36, 222-231. [CrossRef] [PubMed]

3. Creanga, A.A. Maternal Mortality in the United States: A Review of Contemporary Data and Their Limitations. Clin. Obs. Gynecol. 2018, 61, 296-306. [CrossRef]

4. Creanga, A.A.; Syverson, C.; Seed, K.; Callaghan, W.M. Pregnancy-Related Mortality in the United States, 2011-2013. Obs. Gynecol. 2017, 130, 366-373. [CrossRef]

5. Blagoeva Atanasova, V.; Arevalo-Serrano, J.; Antolin Alvarado, E.; Garcia-Tizon Larroca, S. Maternal mortality in Spain and its association with country of origin: Cross-sectional study during the period 1999-2015. BMC Public Health 2018, $18,1171$. [CrossRef]

6. Singh, S.; McGlennan, A.; England, A.; Simons, R. A validation study of the CEMACH recommended modified early obstetric warning system (MEOWS). Anaesthesia 2012, 67, 12-18. [CrossRef]

7. Mhyre, J.M.; D'Oria, R.; Hameed, A.B.; Lappen, J.R.; Holley, S.L.; Hunter, S.K.; Jones, R.L.; King, J.C.; D’Alton, M.E. The maternal early warning criteria: A proposal from the national partnership for maternal safety. Obs. Gynecol. 2014, 124, 782-786. [CrossRef]

8. Isaacs, R.A.; Wee, M.Y.; Bick, D.E.; Beake, S.; Sheppard, Z.A.; Thomas, S.; Hundley, V.; Smith, G.B.; van Teijlingen, E.; Thomas, P.W.; et al. A national survey of obstetric early warning systems in the United Kingdom: Five years on. Anaesthesia 2014, 69, 687-692. [CrossRef]

9. Balk, R.A.; Bone, R.C. The septic syndrome. Definition and clinical implications. Crit Care Clin. 1989, 5, 1-8. 
10. Bone, R.C.; Balk, R.A.; Cerra, F.B.; Dellinger, R.P.; Fein, A.M.; Knaus, W.A.; Schein, R.M.; Sibbald, W.J. Definitions for sepsis and organ failure and guidelines for the use of innovative therapies in sepsis. The ACCP/SCCM Consensus Conference Committee. American College of Chest Physicians/Society of Critical Care Medicine. Chest 1992, 101, 1644-1655. [CrossRef]

11. Singer, M.; Deutschman, C.S.; Seymour, C.W.; Shankar-Hari, M.; Annane, D.; Bauer, M.; Bellomo, R.; Bernard, G.R.; Chiche, J.D.; Coopersmith, C.M.; et al. The Third International Consensus Definitions for Sepsis and Septic Shock (Sepsis-3). JAMA 2016, 315, 801-810. [CrossRef]

12. Evans, L.; Rhodes, A.; Alhazzani, W.; Antonelli, M.; Coopersmith, C.M.; French, C.; Machado, F.R.; McIntyre, L.; Ostermann, M.; Prescott, H.C.; et al. Surviving sepsis campaign: International guidelines for management of sepsis and septic shock 2021. Intensive Care Med. 2021, 47, 1181-1247. [CrossRef]

13. Higgins, R.D.; Saade, G.; Polin, R.A.; Grobman, W.A.; Buhimschi, I.A.; Watterberg, K.; Silver, R.M.; Raju, T.N.K.; Chorioamnionitis Workshop, P. Evaluation and Management of Women and Newborns with a Maternal Diagnosis of Chorioamnionitis: Summary of a Workshop. Obs. Gynecol 2016, 127, 426-436. [CrossRef]

14. Arora, P.; Bagga, R.; Kalra, J.; Kumar, P.; Radhika, S.; Gautam, V. Mean gestation at delivery and histological chorioamnionitis correlates with early-onset neonatal sepsis following expectant management in pPROM. J. Obs. Gynaecol 2015, 35, 235-240. [CrossRef]

15. Navathe, R.; Schoen, C.N.; Heidari, P.; Bachilova, S.; Ward, A.; Tepper, J.; Visintainer, P.; Hoffman, M.K.; Smith, S.; Berghella, V.; et al. Azithromycin vs erythromycin for the management of preterm premature rupture of membranes. Am. J. Obs. Gynecol. 2019, 221, 144.e141-144.e148. [CrossRef]

16. Yudin, M.H.; van Schalkwyk, J.; Eyk, N.V.; Infectious Diseases, C.; Maternal Fetal Medicine, C. Antibiotic therapy in preterm premature rupture of the membranes. J. Obs. Gynaecol. Can. 2009, 31, 863-867. [CrossRef]

17. Gibbs, R.S.; Duff, P. Progress in pathogenesis and management of clinical intraamniotic infection. Am. J. Obs. Gynecol. 1991, 164, 1317-1326. [CrossRef]

18. Kamath, B.D.; Todd, J.K.; Glazner, J.E.; Lezotte, D.; Lynch, A.M. Neonatal outcomes after elective cesarean delivery. Obs. Gynecol. 2009, 113, 1231-1238. [CrossRef]

19. Lappen, J.R.; Keene, M.; Lore, M.; Grobman, W.A.; Gossett, D.R. Existing models fail to predict sepsis in an obstetric population with intrauterine infection. Am. J. Obs. Gynecol. 2010, 203, P573.E1-573.E5. [CrossRef]

20. Kassebaum, N.J.; Bertozzi-Villa, A.; Coggeshall, M.S.; Shackelford, K.A.; Steiner, C.; Heuton, K.R.; Gonzalez-Medina, D.; Barber R.; Huynh, C.; Dicker, D.; et al. Global, regional, and national levels and causes of maternal mortality during 1990-2013: A systematic analysis for the Global Burden of Disease Study 2013. Lancet 2014, 384, 980-1004. [CrossRef]

21. Gaieski, D.F.; Mikkelsen, M.E.; Band, R.A.; Pines, J.M.; Massone, R.; Furia, F.F.; Shofer, F.S.; Goyal, M. Impact of time to antibiotics on survival in patients with severe sepsis or septic shock in whom early goal-directed therapy was initiated in the emergency department. Crit. Care Med. 2010, 38, 1045-1053. [CrossRef]

22. Kramer, H.M.; Schutte, J.M.; Zwart, J.J.; Schuitemaker, N.W.; Steegers, E.A.; van Roosmalen, J. Maternal mortality and severe morbidity from sepsis in the Netherlands. Acta Obs. Gynecol. Scand. 2009, 88, 647-653. [CrossRef]

23. Bauer, M.E.; Bateman, B.T.; Bauer, S.T.; Shanks, A.M.; Mhyre, J.M. Maternal sepsis mortality and morbidity during hospitalization for delivery: Temporal trends and independent associations for severe sepsis. Anesth. Analg. 2013, 117, 944-950. [CrossRef]

24. Karat, C.; Madhivanan, P.; Krupp, K.; Poornima, S.; Jayanthi, N.V.; Suguna, J.S.; Mathai, E. The clinical and microbiological correlates of premature rupture of membranes. Indian J. Med. Microbiol. 2006, 24, 283-285. [CrossRef]

25. Horowitz, E.; Yogev, Y.; Ben-Haroush, A.; Samra, Z.; Feldberg, D.; Kaplan, B. Urine culture at removal of indwelling catheter after cesarean section. Int. J. Gynaecol. Obs. 2004, 85, 276-278. [CrossRef]

26. Atacag, T.; Yayci, E.; Guler, T.; Suer, K.; Yayci, F.; Deren, S.; Cetin, A. Asymptomatic bacteriuria screened by catheterized samples at pregnancy term in women undergoing cesarean delivery. Clin. Exp. Obs. Gynecol. 2015, 42, 590-594.

27. Nasr, A.M.; ElBigawy, A.F.; Abdelamid, A.E.; Al-Khulaidi, S.; Al-Inany, H.G.; Sayed, E.H. Evaluation of the use vs nonuse of urinary catheterization during cesarean delivery: A prospective, multicenter, randomized controlled trial. J. Perinatol. 2009, 29, 416-421. [CrossRef]

28. Bauer, M.E.; Bauer, S.T.; Rajala, B.; MacEachern, M.P.; Polley, L.S.; Childers, D.; Aronoff, D.M. Maternal physiologic parameters in relationship to systemic inflammatory response syndrome criteria: A systematic review and meta-analysis. Obs. Gynecol. 2014, 124, 535-541. [CrossRef]

29. Mackintosh, N.; Watson, K.; Rance, S.; Sandall, J. Value of a modified early obstetric warning system (MEOWS) in managing maternal complications in the peripartum period: An ethnographic study. BMJ Qual. Saf. 2014, 23, 26-34. [CrossRef]

30. Sela, H.Y.; Weiniger, C.F.; Hersch, M.; Smueloff, A.; Laufer, N.; Einav, S. The pregnant motor vehicle accident casualty: Adherence to basic workup and admission guidelines. Ann. Surg. 2011, 254, 346-352. [CrossRef]

31. Blessberger, H.; Kammler, J.; Domanovits, H.; Schlager, O.; Wildner, B.; Azar, D.; Schillinger, M.; Wiesbauer, F.; Steinwender, C. Perioperative beta-blockers for preventing surgery-related mortality and morbidity. Cochrane Database Syst. Rev. 2018, 3, CD004476. [CrossRef] [PubMed]

32. Tawfik, D.S.; Gould, J.B.; Profit, J. Perinatal Risk Factors and Outcome Coding in Clinical and Administrative Databases. Pediatrics 2019, 143, e20181487. [CrossRef] [PubMed] 
33. Greksova, K.; Parrak, V.; Chovancova, D.; Stencl, P.; Oravec, J.; Marsik, L.; Sysak, R.; Fuchs, D.; Peskova, Z.; Borovsky, M. Procalcitonin, neopterin and C-reactive protein in diagnostics of intrauterine infection and preterm delivery. Bratisl. Lek. Listy. 2009, 110, 623-626. [PubMed]

34. Oh, K.J.; Park, K.H.; Kim, S.N.; Jeong, E.H.; Lee, S.Y.; Yoon, H.Y. Predictive value of intra-amniotic and serum markers for inflammatory lesions of preterm placenta. Placenta 2011, 32, 732-736. [CrossRef]

35. Etyang, A.K.; Omuse, G.; Mukaindo, A.M.; Temmerman, M. Maternal inflammatory markers for chorioamnionitis in preterm prelabour rupture of membranes: A systematic review and meta-analysis of diagnostic test accuracy studies. Syst. Rev. 2020, 9 , 141. [CrossRef] 